Canadian

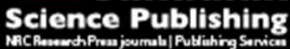

Applied Physiology, Nutrition, and Metabolism Physiologie appliquée, nutrition et métabolisme

\title{
Predicted Versus Measured Thoracic Gas Volumes of Collegiate Athletes Made by the BOD POD Air Displacement Plethysmography System
}

\begin{tabular}{|r|l|}
\hline Journal: & Applied Physiology, Nutrition, and Metabolism \\
\hline Manuscript ID: & apnm-2015-0126.R1 \\
\hline Manuscript Type: & Technical notes \\
\hline Date Submitted by the Author: & 05-May-2015 \\
\hline Complete List of Authors: & Wagner, Dale; Utah State University, HPER \\
\hline Keyword: & $\begin{array}{l}\text { athletes, body composition < body composition, fat mass < body } \\
\text { composition, exercise physiology < exercise }\end{array}$ \\
\hline \multicolumn{2}{|l}{} \\
\hline
\end{tabular}


1

7 Correspondence:

8 Dale R. Wagner, PhD

9 Utah State University

107000 Old Main Hill

11 Logan, UT 84322-7000

12 Phone: 435-797-8253

13 Fax: 435-797-3759

14 Email: dale.wagner@usu.edu
Dale R. Wagner, Ph.D.

Human Movement Science Program, Utah State University, Logan, UT, USA 
15 Abstract

16

Measured (TGVm) and predicted (TGVp) thoracic gas volumes from the BOD POD were

17 compared in 33 lean, university athletes. On average, TGVp (3.529 L) was not significantly

18 different $(p=0.343)$ from TGVm $(3.628 \mathrm{~L})$; however, there was a bias $(r=-.703, p<0.001)$.

19 The difference in $\% \mathrm{BF}$ was within $\pm 2 \% \mathrm{BF}$ for $76 \%$ of the sample, but athletes at the extremes

20 of height should have TGV measured.

21

22 Keywords: body composition, body fat, body volume, lung volumes, precision 


\section{Introduction}

The body composition of athletes is often assessed to identify the optimal level of body fat for elite athletes within a given sport, track changes to monitor the effectiveness of training programs, estimate minimal body weights for those competing in sports with weight classes, and monitor health status to detect and prevent disorders associated with excessively low body fat levels (Heyward and Wagner 2004). The BOD POD, an air displacement plethysmograph, is one of many methods available for estimating body fat percentage $(\% \mathrm{BF})$, and it has steadily increased in popularity since its introduction in 1998. This method is thought to be similar in accuracy to hydrostatic weighing, yet the procedure is quicker and easier for the client and requires minimal technician skill (Heyward and Wagner 2004).

The BOD POD uses the relationship between pressure and volume (Boyle's law) to determine body volume ( $\mathrm{Vb}$ ); body density and \%BF are subsequently calculated. However, $\mathrm{Vb}$ must be corrected for thoracic gas volume (TGV), the quantity of air in the lungs during normal tidal breathing (McCrory et al. 1998). Thoracic gas volume can be measured (TGVm) using a breathing maneuver in the BOD POD, but the machine also offers a predicted TGV (TGVp) that saves time, eliminates the need for disposable breathing tubes and filters, and bypasses a maneuver that some people have difficulty performing successfully. Previous investigators reported good agreement between TGVm and TGVp (Collins and McCarthy 2003; Demerath et al. 2002; McCrory et al. 1998; Peeters 2012), but others found significant differences (Collins et al. 1999; Lockner et al. 2000; Minderico et al. 2008). The purpose of this study was to compare TGVm and TGVp from the BOD POD in lean, collegiate athletes. Tegenkamp et al. (2011) demonstrated that BOD POD-derived \%BF estimates of university athletes could be significantly changed by having the athletes deliberately inhibit full exhalation or inhalation and take shallow 
breaths. However, a comparison of TGVm and TGVp was not included in this research, and this comparison has yet to be made in an athletic population.

\section{Materials and Methods}

\section{Subjects}

Body composition was measured on 45 student-athletes (22 males, 23 females; $20.1 \pm 1.6$ y) from a National Collegiate Athletic Association (NCAA) Division 1 program. The studentathletes were from 7 different sports: football $(n=8)$, golf $(n=7)$, women's gymnastics $(n=9)$, softball $(n=4)$, women's volleyball $(n=1)$, and men's $(n=7)$ and women's tennis $(n=9)$. The study was approved by the institutional review board of Utah State University, and the subjects signed a written informed consent prior to their participation.

\section{Procedures}

The BOD POD (model GS, Cosmed USA, Inc., Concord, CA) was calibrated according to the manufacturer's instructions. Subjects arrived in the lab at least $2 \mathrm{~h}$ after practice, prior to eating, and were instructed to void before being measured. Female subjects wore sports bras, and all subjects wore lycra compression shorts and swim caps, the recommended attire for BOD POD testing (Heyward and Wagner 2004). Height was measured to the nearest $0.1 \mathrm{~cm}$ with a wall-mounted stadiometer (Seca 216, Seca Corp., Ontario, CA). Weight was measured to the nearest $0.001 \mathrm{~kg}$ on the BOD POD scale.

During the BOD POD procedure to obtain $\mathrm{Vb}$, TGVm was determined by a panting or puffing maneuver. For this maneuver, the subject took several normal breaths through a tube while wearing a noseclip. When prompted on the computer screen, the subject gently exhaled 3 times, as if fogging a pair of glasses before cleaning them. The computer prompt for "puffing" coincided with midexhalation and a 3-s period during which the airway through the tube was 
69

70

71

72

73

74

75

76

77

78

79

80

81

82

83

84

85

86

87

88

89

90

91

occluded. A "merit score" is given by the BOD POD following this procedure to indicate if the TGVm is acceptable. Details regarding the calculations to determine TGVm and TGVp from the BOD POD are described elsewhere (McCrory et al. 1998). Body volume and \%BF were recorded for both TGVm and TGVp.

Statistical Analyses

Normality of TGVm and TGVp data were analyzed with the Shapiro-Wilk test. Mean differences between $\mathrm{TGVm}$ and $\mathrm{TGVp}$, as well as $\mathrm{Vb}$ and $\% \mathrm{BF}$ from TGVm and TGVp, were evaluated with paired-samples $t$-tests. Linear regression was used to determine how well predicted variables reflected measured variables. Individual error scores were evaluated with a Bland and Altman (1986) analysis. Statistical significance was established a priori as $p<.05$. All statistical analyses were done with the Statistical Package for Social Sciences (SPSS version 22, IBM, Armonk, NY).

\section{Results}

Measured thoracic gas volume and TGVp were normally distributed $(p=.070)$. Twelve of the subjects (26\%) were unable to achieve acceptable "merit" scores during the puffing maneuver to determine TGVm even after 5 trials; consequently, these subjects were excluded, leaving 33 subjects for data analyses. The mean differences for the variables of interest (TGV, $\mathrm{Vb}$, and \%BF) were not significantly different when TGVm was compared to TGVp (Table 1). The Bland and Altman (1986) analysis (Figure 1) to evaluate individual variability revealed a significant bias $(r=-.703, p<.001)$ such that TGVp was systematically overestimated in subjects with a small TGV and underestimated in those with a large TGV. Nevertheless, the standard error of estimate (SEE) was only $1.3 \% \mathrm{BF}$, and $76 \%$ of the sample were within $\pm 2 \%$ $\mathrm{BF}$ when comparing \%BF derived from TGVp and TGVm. 


\section{Discussion}

The primary finding from this study was that, on average, TGVm and TGVp were not significantly different, and consequently the differences in $\mathrm{Vb}$ and $\% \mathrm{BF}$ between measured and predicted thoracic gas volume trials were not different. This finding coincides with the original investigation of TGVm versus TGVp from the BOD POD by McCrory et al. (1998). These researchers reported no difference between these volumes in 50 adults, and the $\% \mathrm{BF}$ estimation was not affected by TGVp. Other researchers have also reported that mean TGVm and TGVp are similar in adults ranging in age between 16 and $69 \mathrm{y}$ and body mass index (BMI) between 17.8 and $47.2 \mathrm{~kg} \cdot \mathrm{m}^{-2}$ (Collins and McCarthy 2003; Demerath et al., 2002; Peeters 2012), but it appears that there might be significant differences in children (Demerath et al. 2002; Holmes et al. 2011; Lockner et al. 2000).

Despite no mean difference between TGVm and TGVp, there was a significant bias such that those at the extremes of TGV had the highest predictive errors. McCrory et al. (1998) reported no bias in the residual scores of their sample. However, in a study of 85 overweight and obese women, Minderico et al. (2008) reported a systematic bias in TGVp similar in direction and magnitude to the current study of lean, university athletes. The reason for this bias is not apparent, but the similarity to Minderico et al.'s study suggests that it is not population specific. Admittedly, the bias in the present study is created by only a few subjects. If the athlete with the smallest TGV and the four athletes with the largest TGVs are eliminated, the bias disappears. However, their placement at the extremes of TGV is meaningful and indicates a tendency for TGVp from the BOD POD to error toward the mean.

13 Overall, the bias in TGVp had little effect on the estimate of \%BF. This is because only $40 \%$ of the $\mathrm{TGV}$ is used in the calculation of $\mathrm{Vb}$; thus, the impact of even a fairly large error in 
115 TGVp has only a small effect on the estimate of \%BF from the BOD POD (McCrory et al.

116 1998). However, for the five subjects whose TGVm and TGVp values differed by more than 1

$117 \mathrm{~L}$, the difference in $\% \mathrm{BF}$ was greater than $\pm 2 \% \mathrm{BF}$. For most athletes, predicting TGV is not

118 likely to substantially affect their \%BF estimate from the BOD POD; however, for small athletes

119 that might have a $\mathrm{TGVm}<2 \mathrm{~L}$ or large athletes that might have a $\mathrm{TGVm}>5 \mathrm{~L}$, it seems prudent

120 to measure TGV. Height is a variable in the calculation of TGVp (McCrory et al. 1998). In the

121 present study, the shortest athlete $(156 \mathrm{~cm})$ had the most severe overestimation of TGV.

122 Athletes with the largest underestimation of TGV were among the tallest $\left(>185 \mathrm{~cm} ; 80^{\text {th }}\right.$

123 percentile). However, not all short athletes had overestimated TGVp, and not all tall athletes had

124 TGVs that were underestimated.

125 Finally, it was interesting to observe that $26 \%$ of the athletes failed the TGVm procedure.

126 This substantial failure rate further highlights the importance of this research, because for some

127 people TGVp is the only option when being tested in the BOD POD. The majority of these

128 failures were due to high "merit" scores. According to the manufacturer, this suggests that the

129 subject did not maintain a tight seal around the tube while puffing or puffed too hard. Most

130 researchers that have previously compared TGVm to TGVp have not acknowledged or

131 commented on the failure rate to complete the TGVm procedure. Anderson (2007) noted that 3

132 of the 24 adults (13\%) in that study could not complete the procedure, and Lockner et al. (2000)

133 were able to get measurements on only 37 of 54 children aged 10 to $18 \mathrm{y}$, a $31 \%$ failure rate.

134 In summary, this was the first study to compare TGVp and TGVm in an athletic

135 population. There was a systematic bias in TGVp in this sample of university athletes such that

136 TGVp was overestimated in those with the smallest TGV and underestimated in those with the

137 largest TGV. However, this bias affected only a small portion of athletes, and the mean 
138 difference in TGVp was not significantly different from TGVm. Furthermore, errors in TGVp

139 did not substantially impact the estimation of $\% \mathrm{BF}$ for the majority of athletes.

140 Practical Applications

141 When possible, TGVm is recommended when estimating the \%BF of athletes using the

142 BOD POD. Remind athletes to keep a tight seal around the tube and not puff too forcefully in

143 order to achieve an acceptable TGVm. Athletes shorter than $156 \mathrm{~cm}$ or taller than $185 \mathrm{~cm}$ are

144 the ones most susceptible to having a TGVp different from a TGVm. Nevertheless, TGVp will

145 likely provide a valid estimate of TGV for those athletes who are unable to achieve an acceptable

146 merit score during the puffing maneuver. Furthermore, using TGVp rather than TGVm does not

147 substantially change the \%BF estimate for most athletes.

148

149 Acknowledgements

150 Thanks to Dustin Cain for assistance with data collection and the student-athletes for their 151 participation. 


\section{References}

153 Anderson, D.E. 2007. Reliability of air displacement plethysmography. J. Strength Cond. Res. 21(1): 169-172.

Bland, J.M., and Altman, D.G. 1986. Statistical methods for assessing agreement between two methods of clinical measurement. Lancet. 1(8476): 307-310.

Collins, A.L., and McCarthy, H.D. 2003. Evaluation of factors determining the precision of body composition measurements by air displacement plethysmography. Eur. J. Clin. Nutr. 57(6): $770-776$.

160

161

162

163

164

165

166

167

168

169

170

171

172

173

174

Collins, M.A., Millard-Stafford, M.L., Sparling, P.B., Snow, T.K., Rosskopf, L.B., Webb, S.A., et al. 1999. Evaluation of the BOD POD for assessing body fat in collegiate football players. Med. Sci. Sports Exerc. 31(9): 1350-1356.

Demerath, E.W., Guo, S.S., Chumlea, W.C., Towne, B., Roche, A.F., and Siervogel, R.M. 2002. Comparison of percent body fat estimates using air displacement plethysmography and hydrodensitometry in adults and children. Int. J. Obes. 26(3): 389-397.

Heyward, V.H., and Wagner, D.R. 2004. Applied body composition assessment, $2^{\text {nd }}$ ed. Human Kinetics, Champaign, IL.

Holmes, J.C., Gibson, A.L., Cremades, J.G., and Mier, C.M. 2011. Body-density measurement in children: the BOD POD versus hydrodensitometry. Int. J. Sport Nutr. Exerc. Metab. 21(3): $240-247$.

Lockner, D.W., Heyward, V.H., Baumgartner, R.N., and Jenkins, K.A. 2000. Comparison of airdisplacement plethysmography, hydrodensitometry, and dual x-ray absorptiometry for assessing body composition of children 10 to 18 years of age. Ann. N.Y. Acad. Sci. 904: 7278. 
175 McCrory, M.A., Molé, P.A., Gomez, T.D., Dewey, K.G., and Bernauer, E.M. 1998. Body

176 composition by air-displacement plethysmography by using predicted and measured thoracic

177 gas volumes. J. Appl. Physiol. 84(4): 1475-1479.

178 Minderico, C.S., Silva, A.M., Fields, D.A., Branco, T.L., Martins, S.S., Teixeira, P.J., et al. 2008.

179 Changes in thoracic gas volume with air-displacement plethysmography after a weight loss

180 program in overweight and obese women. Eur. J. Clin. Nutr. 62(3): 444-450.

181 Peeters, M.W. 2012. Subject positioning in the BOD POD only marginally affects measurement

182 of body volume and estimation of percent body fat in young adult men. Plos One. 7: e32722.

183 Tegenkamp, M.H., Clark, R.R., Schoeller, D.A., and Landry, G.L. 2011. Effects of covert

184 subject actions on percent body fat by air-displacement plethysmography. J. Strength Cond.

185 Res. 25(7): 2010-2017. 
186 Figure Legend

187 Figure 1. Plot of the TGV residual scores. The solid line is the constant error. The dashed line 188 is the significant relationship $(r=-.703, P<0.001)$ between error scores and the average TGV. 
Table 1. Variables of interest calculated from measured and predicted TGV $(N=33)$

\begin{tabular}{|c|c|c|c|c|c|c|}
\hline Variable & Measured & Predicted & Mean Difference & $P$-value & $R^{2}$ & SEE \\
\hline TGV (L) & $3.628 \pm 0.894$ & $3.529 \pm 0.500$ & $0.099 \pm 0.591$ & 0.343 & 0.613 & 0.566 \\
\hline $\mathrm{Vb}(\mathrm{L})$ & $66.722 \pm 11.163$ & $66.682 \pm 11.129$ & $0.040 \pm 0.237$ & 0.344 & 1.000 & 0.238 \\
\hline$\% \mathrm{BF}$ & $15.2 \pm 6.2$ & $15.0 \pm 6.9$ & $0.2 \pm 1.5$ & 0.361 & 0.958 & 1.303 \\
\hline
\end{tabular}

$\mathrm{TGV}=$ thoracic gas volume; $\mathrm{Vb}=$ body volume; $\% \mathrm{BF}=$ body fat percentage 


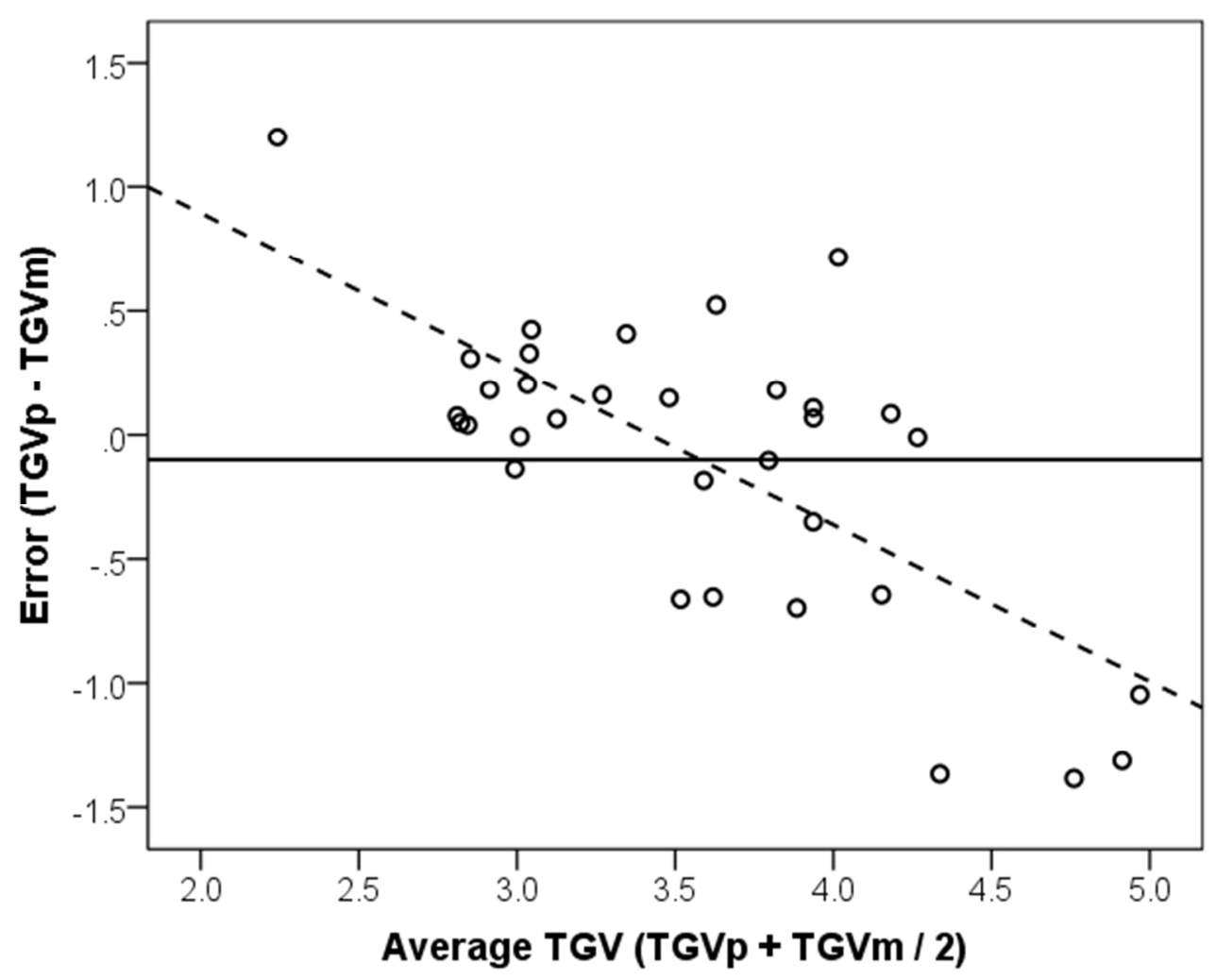

$53 \times 42 \mathrm{~mm}(300 \times 300 \mathrm{DPI})$ 